\title{
Scalable 2D K-SVD Parallel Algorithm for Dictionary Learning on GPUs
}

\author{
Lu He, Timothy Miskell, Rui Liu, Hengyong Yu, Huijuan Xu, Yan Luo \\ Department of Electrical and Computer Engineering, University of Massachusetts Lowell, MA, USA \\ $\{$ Lu_He,Timothy_Miskell,Huijuan_Xu\}@student.uml.edu, \{Rui_Liu,Hengyong_Yu,Yan_Luo\}@uml.edu
}

\begin{abstract}
In recent years, the K-SVD algorithm for dictionary learning has been widely used in the field of image processing. The learning algorithm constructs a dictionary consisting of groups of signal atoms derived from a set of images. The sparse linear combination of the signal atoms are used to construct the best possible match based upon the original images. The myriad applications of K-SVD algorithm include reconstruction, compression, denoising, sparse coding, super resolution, and feature extraction. The K-SVD algorithm is a serial machine learning algorithm whereby each of the signal atoms are trained in succession. All of the signal atoms are updated once within any given iteration. Given that the algorithmic complexity for one iteration is $\mathrm{O}\left(n^{4}\right)$, the training phase of the K-SVD algorithm is timeintensive. In order to increase the speed the K-SVD algorithm and reduce the run-time execution of each iteration, the following paper proposes a parallel version of the $\mathrm{K}$ SVD algorithm and verifies its validity. We design and optimize the parallel algorithm on an Nvidia Titan X GPU by employing three strategies, specifically batches, early stop, and streaming. Experimental results indicate that the parallel algorithm produces a pronounced speedup of $80 \times$ when compared to multi-thread MATLAB implementation of the K-SVD algorithm running on a quad-core CPU.
\end{abstract}

\section{CCS Concepts}

-Computing methodologies $\rightarrow$ Parallel computing methodologies;

\section{Keywords}

GPU; K-SVD; dictionary learning; machine learning; sparse representation; signal denoising

\section{INTRODUCTION}

Over the past few years, unsupervised dictionary learning has come to the forefront for researchers and scholars $[5,15$,

Permission to make digital or hard copies of all or part of this work for personal or classroom use is granted without fee provided that copies are not made or distributed for profit or commercial advantage and that copies bear this notice and the full citation on the first page. Copyrights for components of this work owned by others than ACM must be honored. Abstracting with credit is permitted. To copy otherwise, or republish, to post on servers or to redistribute to lists, requires prior specific permission and/or a fee. Request permissions from permissions@acm.org.

CF'16, May 16-19, 2016, Como, Italy

(c) 2016 ACM. ISBN 978-1-4503-4128-8/16/05 . \$ $\$ 15.00$

DOI: http://dx.doi.org/10.1145/2903150.2903176
30]. The main goal of dictionary learning is to gain knowledge regarding the compact and meaningful signal atoms in order to form an over-complete dictionary from patches of existing images. Afterwards, the sparse linear combination of the learned signal atoms is used to describe existing images and potentially generate matches against future images with markedly similar characteristics. Let us consider image denoising as an example. An over-complete dictionary is generated based upon several patches derived from an image containing noise. The over-complete dictionary expresses the structure of the image and also presents its corresponding noise-free image. After the dictionary is generated, each patch of the noise-free image can be reconstructed according to the sparse linear combination of the signal atoms in the learned dictionary. The applications of such a dictionary include image compression [21, 12], image reconstruction [25, 31 , image denoising $[9,26]$, image deblurring [16] image super-revolution [27], as well as classification [8, 28]. Dictionary learning has been widely used in a number of areas including computer vision $[24,17]$ along with medical image processing $[25,22,20]$.

The objective function of sparse representation in unsupervised dictionary learning is written as

$$
\min _{\mathbf{C}}\|\mathbf{C}\|_{0}
$$

$$
\text { subject to : }\|\mathbf{D C}-\mathbf{X}\|_{F}<\epsilon .
$$

where $\mathbf{C} \in \mathbb{R}^{K \times N}$ is a sparse representation matrix, $\mathbf{D} \in$ $\mathbb{R}^{M \times K}(M<K)$ represents an over-complete dictionary, $\mathbf{X} \in \mathbb{R}^{M \times N}$ is a group of existing image patches, and $\epsilon$ is the infinitesimal error. The $\|\mathbf{C}\|_{0}$ is the $L_{0}$-norm, which denotes the number of non-zero entries in matrix $\mathbf{C}$. The $\|\bullet\|_{F}$ is the Frobenius norm, which is defined as the root square sum of the elements within the matrix. Succinctly, the goal of dictionary learning is to minimize the number of non-zero entries in the sparse representation matrix $\mathbf{C}$ under the constraints that DC is close to $\mathbf{X}$. The difficulty inherent within dictionary learning is that only the image patches, $\mathbf{X}$, are known. Both the dictionary $\mathbf{D}$ and the sparse representation matrix $\mathbf{C}$ are not known a priori.

Solving the problem in Equation 1 is an NP-hard problem [6]. Several papers $[15,10,23,1]$ pursue greedy algorithms as approximate solutions. K-SVD (Singular Value Decomposition) [1] is one of the most representative dictionary learning greedy algorithms [4]. However, the traditional K-SVD algorithm is a serial machine-learning algorithm such that signal atoms in a dictionary are updated in succession. In this case, all the signal atoms are updated once within any given iteration. Training a dictionary is an intensive process because 
the algorithmic complexity of a single iteration is $\mathrm{O}\left(n^{4}\right)$. The aforementioned complexity increases as number of the iterations increases. For example, training a dictionary from a set of image patches derived from a single $512 \times 512$ image would require on the order of tens of hours.

At present it is becoming increasingly popular to repurpose general purpose graphics processing units (GPGPUs) as modified forms of stream processors. A number of timeconsuming applications, including dictionary learning, not only benefit from but require the high performance computing capabilities of GPGPUs. With the aid of general purpose graphics processing units, applications that would normally require several months to execute can be completed in a matter of days .

In this paper, we propose an effective parallel K-SVD algorithm designed for a multi-core architecture. In order to fully utilize the resources of the GPU and to reduce the memory utilization, we design and optimize the implementation of parallel K-SVD algorithm using streaming, batching and early stop strategies on an Nvidia GPU. The main contributions of our work include the following:

- An effective parallel K-SVD algorithm is proposed for a multi-core architecture. The signal atoms in the dictionary are updated in parallel. Experimental results obtained from an image denoising example indicate that constructing the dictionary using a parallel algorithm results in slightly better performance when compared to the serial algorithm.

- We design the parallel K-SVD algorithm on a GPU employing three strategies: early stop, batching, and streaming. The early stop strategy removes the unnecessary calculation performed in the parallel algorithm in order to minimize the utilization of the GPU hardware resources in terms of the computing resources as well as the memory throughput. The streaming strategy strives to ensure that the GPU executes processes in an overlapping manner. Batching builds upon the streaming strategy in that all threads within the batch are designed to run concurrently.

- We implement and optimize the parallel K-SVD algorithm on a GPU. Our design strives to fully utilize all of the available GPU resources. Our parallelized version of the K-SVD algorithm displays a significant amount of speedup when compared to the original serial algorithm.

The following is a description of the organization of this paper. In Section 1 we introduce the relevant background information. Section 2 discusses the related work. Further background information regarding the existing problem as it relates to the K-SVD algorithm is discussed within Section 3. A detailed description of the K-SVD parallel algorithm is given in Section 4. Section 5 describes the design and implementation of the K-SVD algorithm on a set of GPUs. Section 6 presents the experimental results. Section 7 discusses our conclusions along with our intentions for future contributions.

\section{RELATED WORK}

The K-SVD algorithm has continued to gain increasing attention in recent years due to the proliferation of new applications [29, 14, 7, 18]. Several papers have proposed implementations of the K-SVD algorithm as well as the key step orthogonal matching pursuit (OMP) algorithm. For example, [19] proposed an implementation of the K-SVD algorithm and the batch-OMP method. The authors analyzed the time complexity of the K-SVD along with the batch-OMP algorithm, and created an implementation in MATLAB. However, the authors did not employ a parallel analysis and design.

[11] implemented the orthogonal matching pursuit method on a GPU for the purpose of performing compressive sensing, an area related to the field of dictionary learning. The OMP method is a key step within the K-SVD algorithm. The authors analyzed the complexity of each module within the OMP algorithm, determined the bottleneck areas within the modules, and improved the speed of the modules by employing rapid mathematical algorithms, such as Fujimoto's matrix-vector multiplication and Cholesky's inverse-update algorithms. The optimizations focused solely on improving the amount of speedup for each training signal within the loop. However, the K-SVD algorithm requires a significant amount of training signals, implying that employing the methods of parallelization to the training signals normally outside of any given loop will greatly improve performance. Our work will consider both inside and outside parallelism for the OMP method.

[2] proposed a semi-parallel approximate K-SVD algorithm for CT image denoising implemented on a heterogeneous, multicore, distributed memory processor known as the Cell Broadband Engine Architecture. The authors utilized one iteration of Singular Value Decomposition in order to calculate the matrix pseudo-inverse and a pre-computing strategy in order to batch the training signals within the OMP method. For example, they used matrix-matrix multiplication instead of a batch of matrix-vector multiplications. However, the the critical portions of the K-SVD algorithm were still implemented in a serial manner. [13] implemented an approximate K-SVD algorithm using OpenCL on a ATI FirePro V8800 (FireGL V) card from AMD, which is based upon another algorithm derived from [2]. Also, their design does not make use of stream strategy. By comparison, we improve the K-SVD serial algorithm by transforming it into a fully realized parallel algorithm. In addition, our parallelization strategy is wholly unique when compared to previous designs.

\section{BACKGROUND}

In this portion of our work, we introduce the original KSVD algorithm in Section 3.1, and further elucidate the existing problem in Section 3.2. For the sake of consistency in terms of notation, we use bold capital letters for matrices, A, bold lower case letters for vectors, a, capital letters for constants as well as sets, $A$, and lower case letters for variables, $a$. The subscripted vector $\mathbf{a}_{i}$ represents the $i$-th column vector of matrix $\mathbf{A}$, and the superscripted vector $\mathbf{a}_{i}^{T}$ represents the $i$-th row vector of matrix $\mathbf{A}$. The subscripted matrix $\mathbf{A}_{S}$ represents a group of column vectors in matrix A, whereby the indices of the columns belongs to the set $S$. Unless otherwise specified, the notation that follows adheres to the previously mentioned set of rules.

\subsection{The K-SVD Algorithm}

The K-SVD algorithm is used to solve the problem derived 
from Equation 1.

$$
\left\{\begin{array}{l}
\min _{\mathbf{D}, \mathbf{C}}\left\{\|\mathbf{X}-\mathbf{D C}\|_{F}^{2}\right\} \\
\left\|\mathbf{c}_{i}\right\|_{0} \leq T_{0}
\end{array} \quad i=1,2, \cdots, N\right.
$$

where $T_{0}$ is the sparsity level, and $\mathbf{X}, \mathbf{D}$, as well as $\mathbf{C}$ have the same meanings as before with respect to Equation 1. Since the K-SVD algorithm works with two variables $\mathbf{D}, \mathbf{C}$, it is not possible to concurrently compute both $\mathbf{D}$, and $\mathbf{C}$. As a result, it necessary to employ a two-step procedure including a sparse coding stage as well as a dictionary update stage.

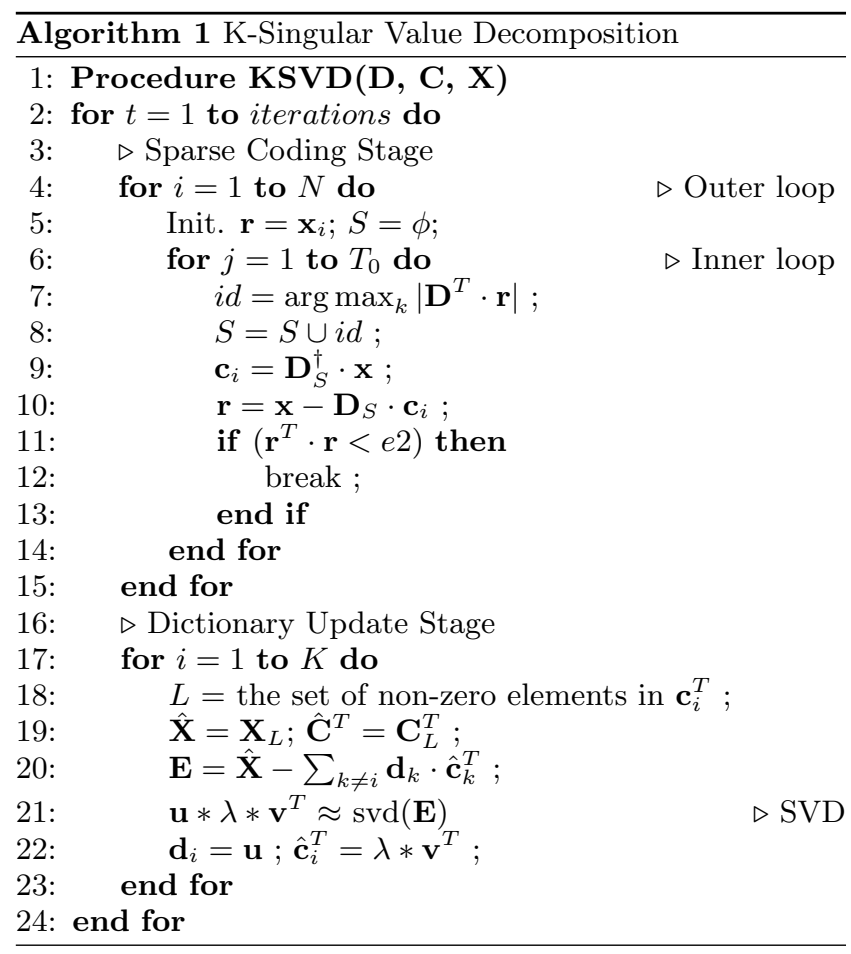

\subsubsection{Sparse Coding Stage}

The sparse coding stage computes the sparse representation vectors $\mathbf{c}_{i}$ of the sample signals $\mathbf{x}_{i}$ based on a fixed dictionary. The equation is written as

$$
\left\{\begin{array}{l}
\min _{\mathbf{c}_{i}}\left\{\left\|\mathbf{x}_{i}-\mathbf{D} \mathbf{c}_{i}\right\|_{2}^{2}\right\} \\
\left\|\mathbf{c}_{i}\right\|_{0} \leq T_{0}
\end{array} \quad i=1,2, \cdots, N\right.
$$

where $\mathbf{x}_{i}$ is the column vector, in other words the signal atom, of the dictionary and $\mathbf{c}_{i}$ is the column vector of the sparse representation matrix. In this stage, the orthogonal matching pursuit (OMP) method is typically used to determine the sparse representations.

The corresponding algorithm of the OMP method is presented in Line 3-15 in Algorithm 1. Subsequently, the inner loop in Line 6 calculates the sparse vector of a sample signal. Line 7 will determine the most recent correlated signal atom in the dictionary as compared with the previous residual. Line 8 combines the most recent correlated signal atom with the previous correlated signal atom in order to form the current signal atom. Line 9 solves the least squares problem based upon the signal atom in Equation 3. Line 10

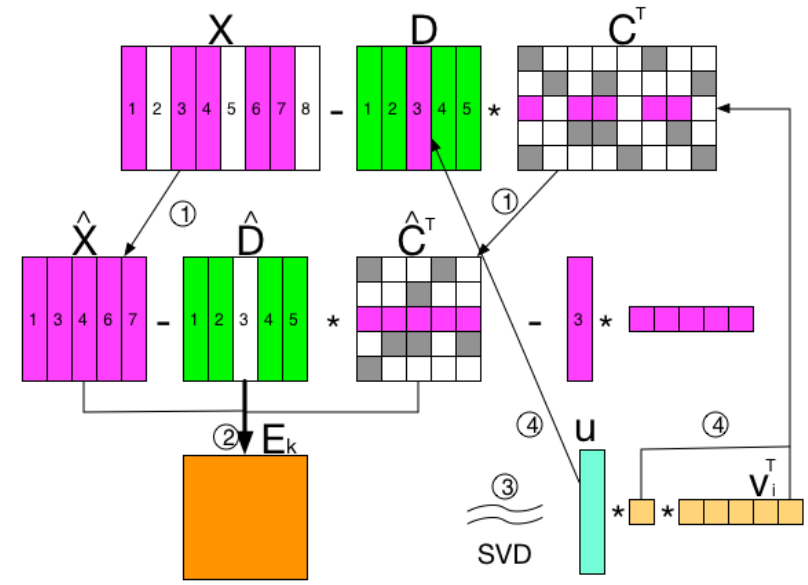

Figure 1: Serial K-SVD algorithm

calculates the residual signal that does not consist of the signal atom. Line 11 determines whether the inner loop should continue. The early stop strategy is important as it prevents the dictionary from learning the noise structure.

\subsubsection{Dictionary Update Stage}

The dictionary update stage refines the dictionary, $\mathbf{D}$, after the sparse representation matrix, $\mathbf{C}$, has been calculated. Returning to the objective function in Equation 2, if the dictionary, $\mathbf{D}$, is divided into a column vector, denoted as $\mathbf{d}_{i}$, and the sparse representation matrix, $\mathbf{C}$, is divided into a row vector, denoted as $\mathbf{c}_{i}^{T}$, the error term can be rewritten as

$$
\|\mathbf{X}-\mathbf{D C}\|_{F}^{2}=\left\|\mathbf{X}-\sum_{i=1}^{N} \mathbf{d}_{i} \cdot \mathbf{c}_{i}^{T}\right\|_{F}^{2}
$$

Supposing that the dictionary and the sparse representation matrix remain unchanged, we can expect $\mathbf{d}_{k}, \mathbf{c}_{k}^{T}$, and the error term to change to

$$
\|\mathbf{X}-\mathbf{D C}\|_{F}^{2}=\left\|\mathbf{X}-\sum_{i \neq k}^{N} \mathbf{d}_{i} \cdot \mathbf{c}_{i}^{T}-\mathbf{d}_{k} \cdot \mathbf{c}_{k}^{T}\right\|_{F}^{2}
$$

Suppose $\mathbf{E}_{\bar{k}}=\mathbf{X}-\sum_{i \neq k}^{N} \mathbf{d}_{i} \cdot \mathbf{c}_{i}^{T}$, where $\bar{k}$ is the set that does not contain $k$. The singular value decomposition method applied to $\mathbf{E}_{\bar{k}}$ can be written as

$$
\mathbf{U} \cdot \boldsymbol{\Lambda} \cdot \mathbf{V}^{T}=\mathbf{E}_{\bar{k}}
$$

where $\mathbf{U}$ and $\mathbf{V}^{T}$ are two orthogonal matrices, and $\boldsymbol{\Lambda}$ is the diagonal matrix whose singular values are sorted in descending order. Since the rank of $\mathbf{d}_{i} \cdot \mathbf{c}_{i}^{T}$ is one, the rank of $\mathbf{E}_{\bar{k}}$ is also one. Thus, the largest singular value $\lambda_{1}$ and its corresponding singular vectors $\mathbf{u}_{1}, \mathbf{v}_{1}^{T}$ are selected to maintain a rank of one as well as to approximate the residual matrix $\mathbf{E}_{\bar{k}}$. The updated signal atom, $\mathbf{d}_{i}$, and the sparse representation vector, $\mathbf{c}_{i}^{T}$, are assigned to $\mathbf{u}_{1}$ and $\lambda_{1} \mathbf{v}_{1}^{T}$. The underlying methodology is presented in Line 16-23 in Algorithm 1. The dictionary update stage refines the signal atoms within the dictionary in a sequential fashion.

The procedural chart for the K-SVD algorithm is shown in Figure 1. Suppose atom 3, colored in magenta, is ready to be updated. The signal and representation matrices are compressed according to the non-zero indices of the third 
row within the representation matrix C. Afterwards atom 3 will be set to zero in step 1 . Step 2 calculates the result of $\mathbf{E}_{k}=\hat{\mathbf{X}}-\hat{\mathbf{D}} \hat{\mathbf{C}}^{T}$. The singular value decomposition is computed and the largest singular value and its corresponding singular vectors are selected to approximate the value of $\mathbf{E}_{k}$ in step 3. Step 4 updates atom 3 and its corresponding representation vector within matrix $\mathbf{C}$.

\subsection{Problems Present with the Existing Algo- rithm and Design}

\subsubsection{Sparse Coding Stage}

Although the sparse representations of the signals can be calculated independently, it still remains a challenge to fully parallelize the sparse coding stage. In this case, the sparse coding stage can be implemented using two different levels of parallelization. The first level is the outer loop, presented in Line 4, which calculates the sparse representation vector of the independent sample signals. The second level is the inner loop, presented in Line 11, which performs the matrix operations. A parallel implementation of the inner loop can easily be implemented with the aid of a cuBLAS library along with a set of CUDA kernels. The outer loop can be implemented in a multi-core architecture without the need for complicated data communication. However, the parallelization of the outer loop along with the matrix operations is significantly more complex.

Another problem introduced within the sparse coding stage is the early stop condition, which is presented in Line 11. Specifically, different image patches have different sparse representation vectors. Furthermore, some sparse representations require less iterations, while others may require more as a result of an early stop. If the outer loop is implemented in parallel, a significant amount of computational resources will be wasted on errant calculations.

To address these issues, we apply a batching strategy to calculate the sparse coding of a group of sample signals concurrently, and apply a streaming strategy to different groups in order to overlap resource utilization. In addition, an early stop strategy is employed to reduce the consumption of computing resources, and further improve the amount of speedup.

\subsubsection{Dictionary Update Stage}

An obvious problem with the K-SVD algorithm is that it is a serial algorithm. In this case, for each iteration, at the moment when each signal atom is obtained, the dictionary matrix must be updated. Although the matrix-matrix multiplication along with the SVD in the dictionary update stage can be implemented in parallel, the GPU's computing resources will remain under utilized. Therefore, a fully parallel K-SVD algorithm is imperative when the dictionary is updated in parallel.

When the serial algorithm is converted into parallel algorithm, two different conditions must be considered. One is the "same position" condition, presented in the left of Figure 2. In this case, the representation vectors, $\mathbf{c}_{i}^{T}$ and $\mathbf{c}_{j}^{T}$, have the same non-zero positions, however, the values within each of the non-zero positions are distinct. The "same position" condition results in a set of superfluous signal atoms within the dictionary. The other is the "different position" condition, presented in the right of Figure 2, which presents distinct position patterns for each of the representation vec-

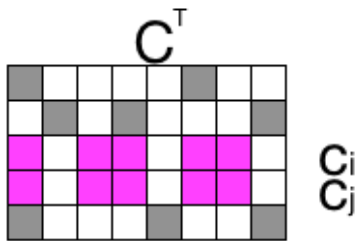

Same Position

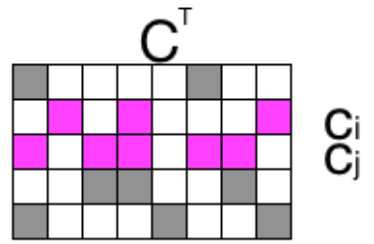

Different Position
Figure 2: The two conditions present within the parallel K-SVD algorithm

tors, $\mathbf{c}_{i}^{T}$ and $\mathbf{c}_{j}^{T}$. The "different position" condition results in a loss of the sparse attribution of the representation vectors.

To address these problems, we propose a fully parallelized K-SVD algorithm, which will update the dictionary in a parallel manner. Moreover, our method solves the problems illustrated within Figure 2. This algorithm has the potential to significantly improve the level of speedup. Furthermore, the experimental results indicate that the algorithm produces improved results in the field of image denoising, as presented in detail within Section 6.2. Streaming and batching strategies are employed as part of the parallel KSVD algorithm. Further details are presented within Section 4.2 .

\section{K-SVD PARALLEL ALGORITHM}

In order to solve the problems listed in Section 3.2, we propose a parallel K-SVD algorithm well suited for a GPU implementation.

\subsection{Sparse Coding Stage}

The improved parallel OMP algorithm is shown in Algorithm 2 . In this case, the sparsity loop has now been moved to the outside. The remaining loops in the serial algorithm are now calculated in parallel. The flag vector $\mathbf{f}$ is used to record an early stop event for each thread. The set $F$ contains the current signals that are awaiting further calculation. Line 5-8 presents the parallel algorithm that is used to find the sparse representation. Line 9 handles the early stop condition. Specifically, if the squared error of a given thread is less than a specified threshold a flag will be set. Subsequently, the algorithm will remove any signals that have stopped early from set $F$ and continue calculating the next representation value. As a result, the design has the potential to remove unnecessary calculations in order to further improve performance.

\subsection{Dictionary Update Stage}

In the dictionary update stage, we first illustrate the feasibility of the parallel algorithm. The object of the parallel K-SVD algorithm is to calculate the signal atoms in parallel, after which the dictionary is updated in parallel. Each of the problems described in Section 3.2.2 will be solved in turn within the paragraphs that follow.

\subsubsection{The "Same Position" Problem}

With reference to Equation 4, if some of the signal atoms, $b$, are batched into a group, denoted as set $B$, the residual 


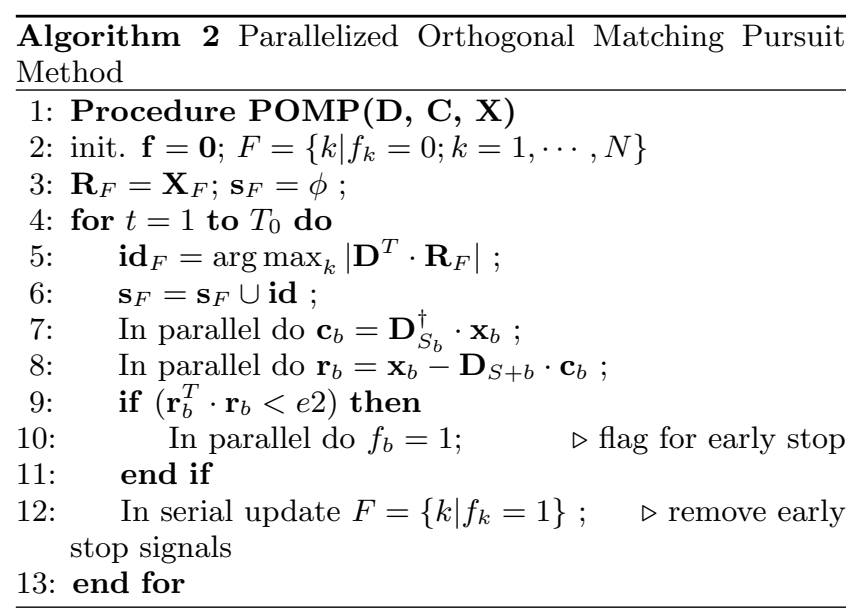

term can be rewritten as

$$
\|\mathbf{X}-\mathbf{D C}\|_{F}^{2}=\left\|\mathbf{X}-\sum_{i \notin B}^{N} \mathbf{d}_{i} \cdot \mathbf{c}_{i}^{T}-\sum_{i \in B} \mathbf{d}_{i} \cdot \mathbf{c}_{i}^{T}\right\|_{F}^{2}
$$

Suppose that $\mathbf{E}_{\bar{B}}=\mathbf{X}-\sum_{i \notin B} \mathbf{d}_{i} \cdot \mathbf{c}_{i}^{T}$. If we apply singular value decomposition to Equation 7 , the residual matrix $\mathbf{E}_{\bar{B}}$ can be decomposed into two orthogonal matrices, $\mathbf{U}$ and $\mathbf{V}^{T}$, and one diagonal matrix, $\boldsymbol{\Lambda}$.

Given that the rank of $\sum_{i \in B} \mathbf{d}_{i} \cdot \mathbf{c}_{i}^{T}$ is given by $b$, the largest $b$ singular values, $\boldsymbol{\Lambda}_{b}$, and their corresponding singular vectors, $\mathbf{U}_{b}$ along with $\mathbf{V}_{b}^{T}$, can be selected in order to approximate $\mathbf{E}_{\bar{B}}$. The approximate error is given by

$$
\left\|\mathbf{E}-\mathbf{U}_{b} \boldsymbol{\Lambda}_{b} \mathbf{V}_{b}^{T}\right\|_{F} \leq\left\|\mathbf{E}-\mathbf{u}_{1} \lambda_{1} \mathbf{v}_{1}^{T}\right\|_{F}
$$

In addition, the more singular values we use, the better the approximation becomes. Afterwards, the singular vectors are updated based upon the signal atoms and the representation vectors. The overall procedure is shown in Figure 3 .

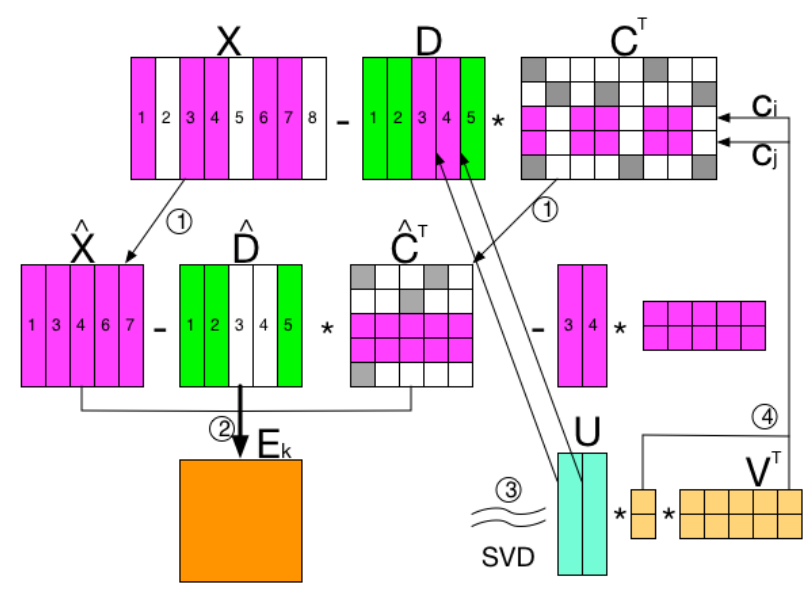

Figure 3: The "Same Position" Problem

\subsubsection{The "Different Position" Problem}

The "Same Position" problem presented previously allows updating each signal atom and representation vector according to the singular vectors $\mathbf{U}_{b}$ and $\mathbf{V}_{b}^{T}$. However, with re- spect to the "Different Position" problem, it is no longer possible to leverage equation 7 . Specifically, the group of signals are reliant upon the majority of the signal atoms that are contained within the dictionary. At this point, the current representations will remain sparse. However, after the singular value decomposition of the residual matrix $\mathbf{E}_{\bar{B}}$, the singular vectors will no longer be sparse. As shown within Figure 4, the aforementioned singular vectors will become the representation vector in the next iteration.

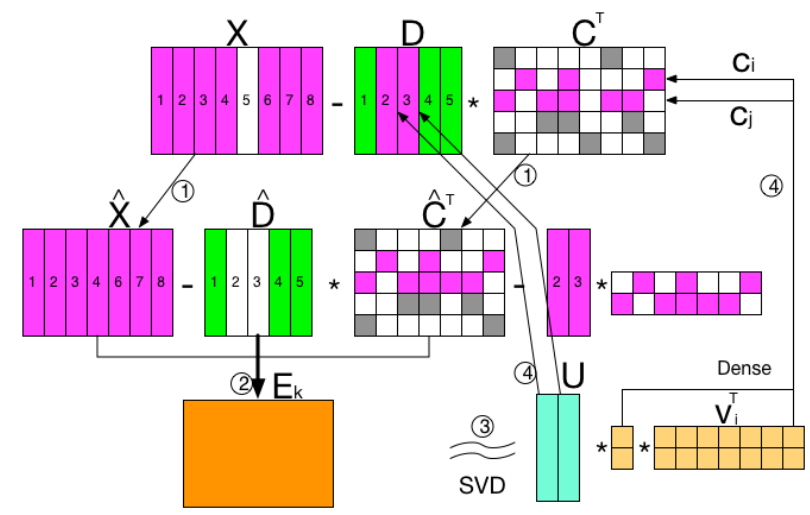

Figure 4: The "Different Position" Problem

In order to parallelize this "Different Position" problem while maintaining a sparse representation matrix, we revise Equation 5 to become

$$
\|\mathbf{X}-\mathbf{D C}\|_{F}^{2}=\frac{\sum_{i=k_{1}}^{k_{b}}\left\|\mathbf{E}_{i} \cdot \mathbf{c}_{i}^{T}-\mathbf{d}_{k} \cdot \mathbf{c}_{k}^{T}\right\|_{F}^{2}}{b}
$$

For each residual matrix after the first order SVD of $\mathbf{E}_{i}$, the signal atom and sparse representations of the batch signals will have been generated. As result, we are able to maintain a sparse representation by preventing the possibility of an overlap.

\subsubsection{The Improved Parallel Algorithm}

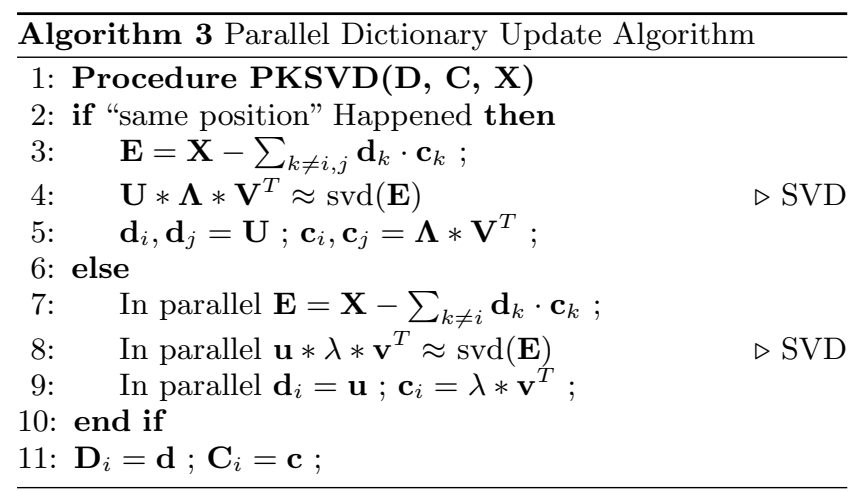

The parallel K-SVD algorithm is shown in Algorithm 3. The algorithm will check whether a "Same Position" condition was detected within any of the sparse representation matrices. If the condition occurred, the algorithm will calculate one SVD in order to update $\mathbf{d}_{i}$, and $\mathbf{d}_{j}$. If the condition did not occur, the algorithm will calculate the SVD for the residual of each of the signal atoms within the dictionary 
and then perform an update. In this case, all of the signal atoms can be calculated in parallel and then updated within the dictionary.

\section{PARALLEL IMPLEMENTATION}

In this section, we first implement the serial K-SVD algorithm but parallelize the matrix operations within the inner loop. Afterwards, we apply our streaming and batching strategies in order to parallelize the outer loop. In addition, we use an early stop strategy in order to reduce the possibility of performing unnecessary calculations.

\subsection{Baseline: Inner Loop Parallelization}

The inner loop parallelization consists of the matrix-vector multiplication, the argument of the maximum, the pseudoinverse of a matrix, vector normalization, as well as the singular value decomposition performed during the dictionary refinement phase. With the exception of the singular value decomposition and the pseudo-inverse of a matrix, the majority of the operations can be implemented with the aid of the cuBLAS library provided by Nvidia. The pseudoinverse of a dictionary, $\mathbf{D}^{\dagger}$, which is equal to $\mathbf{A}^{T}\left(\mathbf{A} \mathbf{A}^{T}\right)^{-1}$, can also be implemented with the aid of the cuBLAS interface. For singular value decomposition, we use the block power method mentioned in [3].

The inner parallelization design has a speedup of $4 \times$ when compared to the MATLAB implementation of the K-SVD algorithm. The minimal level of speedup is primarily the result of the serial design of the outer loop. Furthermore, we observe that all of the calculation matrices were small. Although some of the matrices were large, they were subdivided into smaller columns or row vectors for the ensuing calculations. Operations performed on the smaller matrices within the serial outer loop resulted in two problems. The first problem is that the computing resources of the GPU were not fully utilized. The second problem is that the GPU had to launch thousands of GPU kernels. When the GPU kernels were small, the launch time become a significant issue.

As a result, in order to fully utilize the GPU's computing resources, we parallelize the outer loop with the aid of the batching and streaming strategies described within the next subsection.

\subsection{Streaming and Batching Strategy}

The streaming and batching parallel design is shown in Figure 5. The task creates several streams, each of which may work in an overlapping or concurrent fashion depending on the utilization of the computational resources or the copying engine. Each of the streaming instances will run on the batching kernels, with each of the batching kernels running concurrently.

CUDA streams are used to overlap some of the computations performed within the independent tasks. Furthermore, the batching technology allows for the simultaneous execution of multiple kernels, effectively reducing the kernel launch time and in turn improving performance. In order to launch a plethora of kernels simultaneously we create several streams, whereby each stream can execute a group of kernels.

The cuBLAS library includes interfaces with streaming and batching features, such as matrix-matrix multiplication along with the inverse of square matrices. For functions that

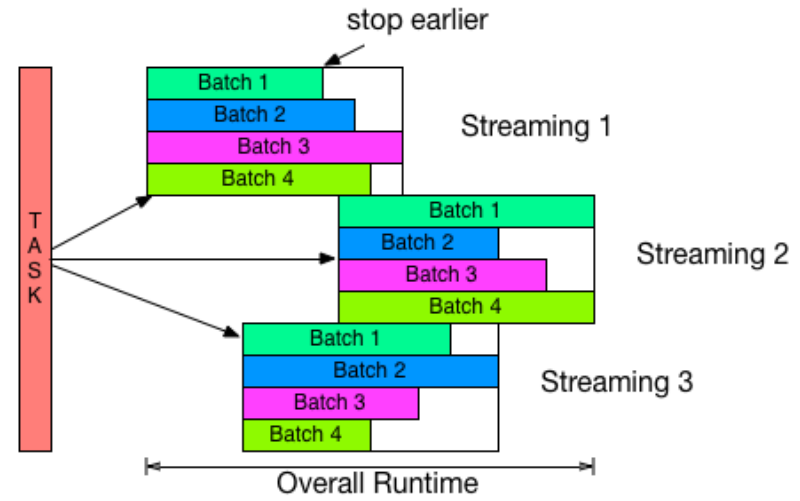

Figure 5: Streaming and Batching Architecture

require the batching interface, it is no longer necessary to directly implement the kernel.

However, some of the interfaces do not provide batching features, such as vector normalization. In this case, we design the batching kernels so that one kernel can perform a group of calculations. When combined with streaming technology, our design can achieve up to a $70 \times$ speedup as compared with the initial design. Further details are provided in Section 6.1.

\subsection{Early Stop Strategy}

During the sparse coding phase, most of the signals can not reach the sparsity level, $T_{0}$, as shown in Figure 5 . As observed, the average sparsity portion is between $22 \%-70 \%$ of the sparsity level $T_{0}$. Thus, our early stop design is a critical component for realizing performance improvements.

In this case, whenever some of the batches stop early, we collect any unfinished tasks into a block and then move forward with the procedure. However, moving data is an expensive operation for the GPU. As a result, we avoid moving data in order to improve the speedup of the early stop design. Instead, we define a pointer array that points to each of the signals along with their corresponding sparse representation vectors. Whenever a task completes, we simply remove the pointer for the completed task and in turn reduce the size of the pointer array. The costs associated with updating the pointer array are significantly less than the costs associated with moving large blocks of data.

\section{EXPERIMENTS}

We demonstrate our parallelization of the K-SVD algorithm using a number of real-world images. The target platform consisted of a quad-core Intel(R) Xeon(R) W3550 processor $(3.07 \mathrm{GHz})$ with a Nvidia GeForce GTX TITAN X $(1.08 \mathrm{GHz})$. Furthermore, the experiment utilized MATLAB version R2014a. The CPU processor included $32 \mathrm{~KB}$ of L1 data cache per core, $32 \mathrm{~KB}$ of L1 instruction cache per core, $256 \mathrm{~KB}$ of L2 cache per core, and $8 \mathrm{MB}$ of L3 cache, which was shared by all cores. In addition, the experiment utilized 12 GB of physical memory. The GPU had 3072 CUDA Cores, $3 \mathrm{MB}$ of L2 cache and $12 \mathrm{~GB}$ of global memory.

\subsection{Overall Performance}

In this section, we focus on the speedup of our parallel algorithm executing on the GPU over the serial algorithm 


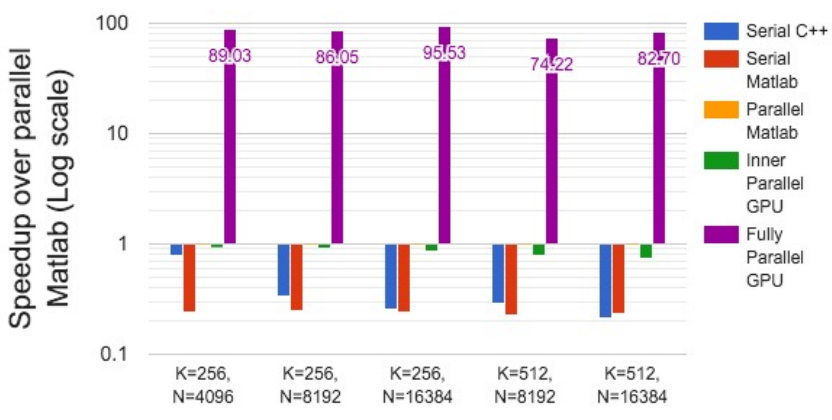

Figure 6: Speedup compared to Parallel MATLAB (Log scale)

implemented on C, serial MATLAB and multicore MATLAB (2014a). For the experiment, the image batches were configured to a nominal setting of $M=8 * 8$. The noise level, $\sigma$, was 1 , and the sparsity level, $T_{0}$ was 32 . The training batches, $N$, and the number of dictionaries, $K$, were variables. The average speedup is shown in Figure 6. The figure compares five different designs. The "Serial $\mathrm{C}++$ " and "Serial MATLAB" portions represent the serial $\mathrm{C}++$ and MATLAB implementation. "Parallel MATLAB" portions represents the multi-thread MATLAB implementation on a quad-core 8-thread CPU. The "Inner" portion represents only the inner loop in Algorithm 1, which in our case was implemented in parallel. The "Fully parallel GPU" portion represents the fully parallel implementation of the algorithm, complete with the batching, streaming and early stop strategies. From the figure, we can see that the fully parallel implementation displays approximately a $80 \times$ speedup when compared to the multi-thread MATLAB implementation.

\begin{tabular}{|r|c|c|c|c|}
\hline Noise Level & \multicolumn{2}{|c|}{ Parallel K-SVD } & \multicolumn{2}{c|}{ Serial K-SVD } \\
\hline & SNR & Sparsity & SNR & Sparsity \\
\hline \hline$\sigma=1$ & 48.67 & $34.76 \%$ & 48.67 & $34.79 \%$ \\
\hline$\sigma=5$ & 37.96 & $10.97 \%$ & 37.95 & $11.0 \%$ \\
\hline$\sigma=10$ & 34.23 & $5.13 \%$ & 34.20 & $5.14 \%$ \\
\hline
\end{tabular}

Table 1: SNR of the recovery image and the sparsity of the representations for the parallel and serial algorithms.

\subsection{Effectivity comparison between the serial and parallel algorithms}

In this section, we use image denoising as an example to show the effectivity of the dictionary learned by both the serial and parallel K-SVD algorithms. We select 50 medical lung images as our dataset. For each image, we apply Gaussian additive noise with a mean of zero, a standard deviation of one, $\mathbf{G}$, onto the original image $\mathbf{I}$ as

$$
\hat{\mathbf{I}}=\mathbf{I}+\sigma * \mathbf{G} \text {. }
$$

The level of Gaussian additive noise is given by $\sigma$. We use the signal to noise ratio (SNR) along with the sparsity of the representation matrix to evaluate the learned dictionary. The SNR is calculated as

$$
S N R=10 * \log _{10}(\text { Image } / \text { Noise }) .
$$

In this case, a comparison is made between the recovery image level and the background noise level. The higher the signal to noise ratio, the better the recovered image. The sparsity is defined as the ratio of non-zero entries to the total number of entries within the representation matrix. Low sparsity is usually an indicator that a suitable dictionary has been generated.

We randomly select 4096 small image patches from each medical image in order to train the dictionary, with the number of training iterations set to 20 . The average results for different noise levels, $\sigma$, are shown in Table 1 . We find that the SNR for the parallel K-SVD algorithm is slightly higher than that for the serial K-SVD algorithm. Furthermore, the sparsity of the parallel K-SVD algorithm is slightly lower than the serial K-SVD algorithm, particularly as the noise level increases.

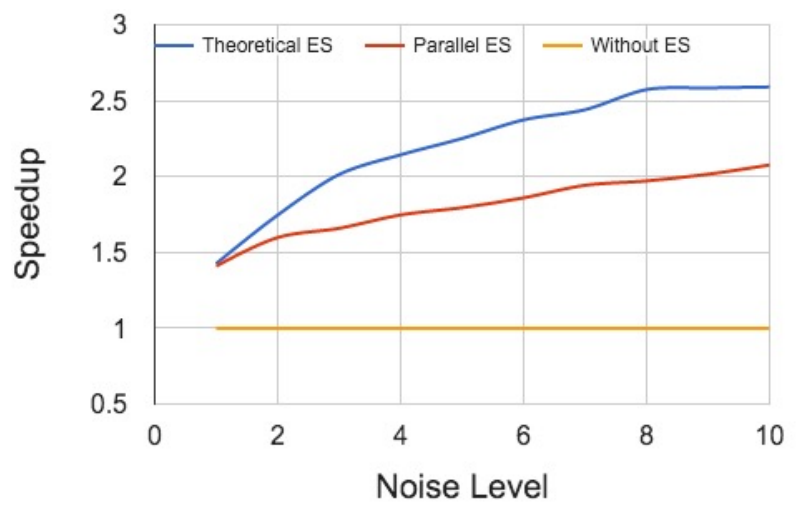

Figure 7: Speedup of the observed and theoretical values

\subsection{Early Stop Evaluation}

In this section, we will compare the performance of the parallel algorithm with and without the early stop design. For the experiment, the sparsity level $T_{0}$ was set to 32 , the number of training batches, $N$, was set to 4096 , and the number of signal atoms within dictionary was 256. As we noticed in Table 1 , the noise level, $\sigma$, affects the sparsity of the representation matrix, and in turn the early stop portion of the parallel algorithm. As a result we evaluate the relationship between the noise level and the theoretical speedup as well as the parallel implementation speedup. The theoretical speedup is defined as the ratio of the maximal number of the sparsity to the average number of the sparsity. The parallel speedup is calculated by the run-time of the early stop version divided by the run-time of the version for which early stop is not present. The results are shown in Figure 7. By observation, when the noise level, $\sigma$, is 1 , the theoretical speedup approximates the actual speedup. This is because when the noise level is small, it requires more signal atoms to represent the sample signals. In this case, the execution time for the GPU is long enough that the execution time for the CPU can be ignored. When the noise level becomes large enough, less signal atoms are required to represent the sample signals. So that the theoretical speedup increases rapidly while the observed speedup only increases by a factor of one half. This is because the execution time on the GPU reduced significantly, while the control time on the CPU takes around $1 / 3$ of the total execution time, which in turn affects the actual speedup. 


\section{CONCLUSIONS}

We propose a parallel implementation of the K-SVD algorithm and execute it on a GPU. With our design, the implementation displayed a speedup of up to $350 \times$ when compared to a multi-core MATLAB implementation. In addition, the experimental results indicate that our parallel algorithm is slightly better than the serial algorithm in the field of image denoising. In the future, we plan to apply our parallel K-SVD algorithm in the field of medical image reconstruction.

\section{ACKNOWLEDGMENTS*}

This work is supported in part by the National Science Foundation. (No. 1547428, No. 1541434, No. 1440737 and No. 1450996) and a gift from Intel Corporation (Grant ID 23903231).

\section{REFERENCES}

[1] M. Aharon, M. Elad, and A. Bruckstein. K-svd: An algorithm for designing overcomplete dictionaries for sparse representation. Signal Processing, IEEE Transactions on, 54(11):4311-4322, 2006.

[2] D. Bartuschat, A. Borsdorf, H. Köstler, R. Rubinstein, and M. Stürmer. A parallel k-svd implementation for ct image denoising. Fridrich-Alexander University, Erlangen, 2009.

[3] A. Bentbib and A. Kanber. Block power method for svd decomposition.

[4] A. M. Bruckstein, D. L. Donoho, and M. Elad. From sparse solutions of systems of equations to sparse modeling of signals and images. SIAM review, 51(1):34-81, 2009.

[5] E. J. Candes, J. Romberg, and T. Tao. Robust uncertainty principles: Exact signal reconstruction from highly incomplete frequency information. IEEE Trans. Inf. Theor., 52(2):489-509, Feb. 2006.

[6] G. Davis, S. Mallat, and M. Avellaneda. Adaptive greedy approximations. Constructive approximation, 13(1):57-98, 1997.

[7] X. Deng and Z. Liu. Image denoising based on steepest descent omp and k-svd. In Signal Processing, Communications and Computing (ICSPCC), 2015 IEEE International Conference on, pages 1-5. IEEE, 2015.

[8] W. Dong, X. Li, D. Zhang, and G. Shi. Sparsity-based image denoising via dictionary learning and structural clustering. In Computer Vision and Pattern Recognition (CVPR), 2011 IEEE Conference on, pages 457-464, June 2011.

[9] M. Elad and M. Aharon. Image denoising via sparse and redundant representations over learned dictionaries. Image Processing, IEEE Transactions on, 15(12):3736-3745, 2006.

[10] K. Engan, K. Skretting, and J. H. Husøy. Family of iterative ls-based dictionary learning algorithms, ils-dla, for sparse signal representation. Digital Signal Processing, 17(1):32-49, 2007.

[11] Y. Fang, L. Chen, J. Wu, and B. Huang. Gpu implementation of orthogonal matching pursuit for compressive sensing. In Parallel and Distributed Systems (ICPADS), 2011 IEEE 17th International Conference on, pages 1044-1047. IEEE, 2011.

[12] I. Horev, O. Bryt, and R. Rubinstein. Adaptive image compression using sparse dictionaries. In Systems, Signals and Image Processing (IWSSIP), 2012 19th International Conference on, pages 592-595. IEEE, 2012.

[13] P. Irofti and B. Dumitrescu. Gpu parallel implementation of the approximate k-svd algorithm using opencl. In Signal Processing Conference (EUSIPCO), 2014 Proceedings of the 22nd European, pages 271-275. IEEE, 2014.

[14] Z. Jiang, Z. Lin, and L. S. Davis. Learning a discriminative dictionary for sparse coding via label consistent k-svd. In
Computer Vision and Pattern Recognition (CVPR), 2011 IEEE Conference on, pages 1697-1704. IEEE, 2011.

[15] K. Kreutz-Delgado, J. F. Murray, B. D. Rao, K. Engan, T.-W. Lee, and T. J. Sejnowski. Dictionary learning algorithms for sparse representation. Neural computation, 15(2):349-396, 2003.

[16] L. Ma, L. Moisan, J. Yu, and T. Zeng. A dictionary learning approach for poisson image deblurring. Medical Imaging, IEEE Transactions on, 32(7):1277-1289, 2013.

[17] I. Ramirez, P. Sprechmann, and G. Sapiro. Classification and clustering via dictionary learning with structured incoherence and shared features. In Computer Vision and Pattern Recognition (CVPR), 2010 IEEE Conference on, pages 3501-3508. IEEE, 2010.

[18] W. Ruangsang and S. Aramvith. Super-resolution for hd to $4 \mathrm{k}$ using analysis k-svd dictionary and adaptive elastic-net. In Digital Signal Processing (DSP), 2015 IEEE International Conference on, pages 1076-1080. IEEE, 2015.

[19] R. Rubinstein, M. Zibulevsky, and M. Elad. Efficient implementation of the k-svd algorithm and the batch-omp method. Department of Computer Science, Technion, Israel, Tech. Rep, 2008.

[20] L. Shi, X. Yin, L. Zhang, B. Yang, J. Zhan, Y. Chen, H. Shu, et al. Improving low-dose brain perfusion computed tomography using $3 \mathrm{~d}$ dictionary learning based processing. Journal of Medical Imaging and Health Informatics, 5(7):1494-1498, 2015.

[21] K. Skretting and K. Engan. Image compression using learned dictionaries by rls-dla and compared with k-svd. In Acoustics, Speech and Signal Processing (ICASSP), 2011 IEEE International Conference on, pages 1517-1520. IEEE, 2011.

[22] S. Tan, Y. Zhang, G. Wang, X. Mou, G. Cao, Z. Wu, and H. Yu. Tensor-based dictionary learning for dynamic tomographic reconstruction. Physics in medicine and biology, 60(7):2803, 2015.

[23] J. Tropp, A. C. Gilbert, et al. Signal recovery from random measurements via orthogonal matching pursuit. Information Theory, IEEE Transactions on, 53(12):4655-4666, 2007.

[24] J. Wright, Y. Ma, J. Mairal, G. Sapiro, T. S. Huang, and S. Yan. Sparse representation for computer vision and pattern recognition. Proceedings of the IEEE, 98(6):1031-1044, 2010.

[25] Q. Xu, H. Yu, X. Mou, L. Zhang, J. Hsieh, and G. Wang. Low-dose $\mathrm{x}$-ray ct reconstruction via dictionary learning. Medical Imaging, IEEE Transactions on, 31(9):1682-1697, 2012.

[26] R. Yan, L. Shao, and Y. Liu. Nonlocal hierarchical dictionary learning using wavelets for image denoising. Image Processing, IEEE Transactions on, 22(12):4689-4698, 2013.

[27] J. Yang, J. Wright, T. S. Huang, and Y. Ma. Image super-resolution via sparse representation. Image Processing, IEEE Transactions on, 19(11):2861-2873, 2010.

[28] M. Yang, D. Dai, L. Shen, and L. Van Gool. Latent dictionary learning for sparse representation based classification. In Computer Vision and Pattern Recognition (CVPR), 2014 IEEE Conference on, pages 4138-4145, June 2014.

[29] Q. Zhang and B. Li. Discriminative k-svd for dictionary learning in face recognition. In Computer Vision and Pattern Recognition (CVPR), 2010 IEEE Conference on, pages 2691-2698. IEEE, 2010.

[30] Z. Zhang, Y. Xu, J. Yang, X. Li, and D. Zhang. A survey of sparse representation: Algorithms and applications. Access, IEEE, 3:490-530, 2015.

[31] B. Zhao, H. Ding, Y. Lu, G. Wang, J. Zhao, and S. Molloi. Dual-dictionary learning-based iterative image reconstruction for spectral computed tomography application. Physics in medicine and biology, 57(24):8217, 2012. 